UCRL-CR-125905

B317413

Preparation-Induced Errors in EPR Dosimetry of Enamel: Pre- and Post-crushing Sensitivity

E.H. Haskell, R.B. Hayes, G.H. Kenner

January 1996

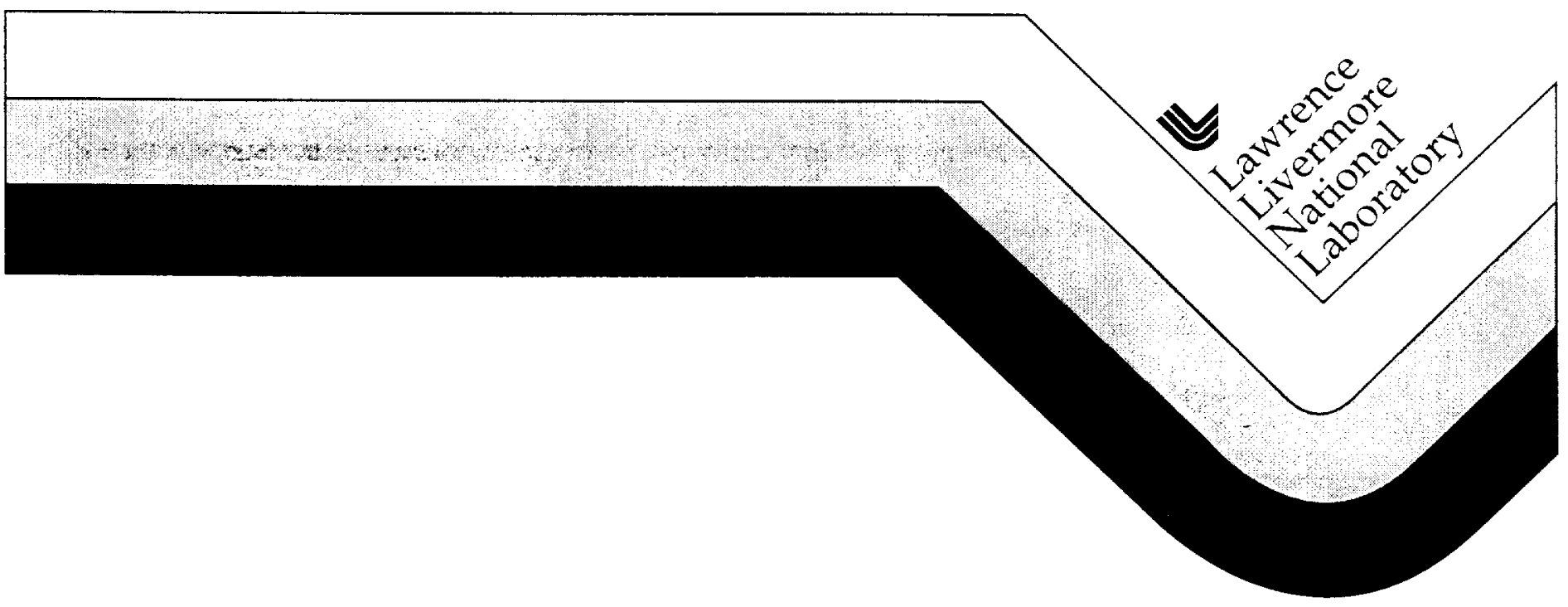




\section{DISCLAIMER}

This document was prepared as an account of work sponsored by an agency of the United States Government. Neither the United States Government nor the University of California nor any of their employees, makes any warranty, express or implied, or assumes any legal liability or responsibility for the accuracy, completeness, or usefulness of any information, apparatus, product, or process disclosed, or represents that its use would not infringe privately owned rights. Reference herein to any specific commercial product, process, or service by trade name, trademark, manufacturer, or otherwise, does not necessarily constitute or imply its endorsement, recommendation, or favoring by the United States Government or the University of California. The views and opinions of authors expressed herein do not necessarily state or reflect those of the United States Government or the University of California, and shall not be used for advertising or product endorsement purposes.

Work performed under the auspices of the U.S. Department of Energy by Lawrence Livermore National Laboratory under Contract W-7405-ENG-48. 


\title{
Preparation-induced Errors in EPR Dosimetry of Enamel: Pre- and Post-crushing Sensitivity
}

\author{
E.H. HASKELL*, R.B. HAYES and G.H. KENNER \\ Division of Radiobiology, College of Medicine, University of Utah, Salt Lake City. UT 84112. \\ U.S.A.
}

\begin{abstract}
Polyakuv er al. (1995) showed errors in dose estimation as a function of grain size for enamel gaans given betia irradiation after crushing. We tested the effect of gamma irradiation applied to the specimers before and after crushing.

We confirmed Polyakov's observations and found that post-crushing irradiation altered the slope of the dose-response curve of the hydroxyapatite signal and produced a grain-size-dependent offset. No changes in the slope of the dose-response curve were seen in enamel caps irradiated whoke before crushing. Copyright $f 1996$ Elsevier Science Lid
\end{abstract}

\section{Introduction}

Tooth enamel of various origins is a promising material for dating geological materials and retrospective accident dosimetry (Grün et al., 1990; Ikeya et al., 1986; Ishii and Ikeya, 1990; Nishiwaki and Shimano, 1990: Pass and Aldrich, 1985; Rink and Schwarcz, 1994: Shimano et al., 1989; Stringer et al., 1989; Tatsumi-Miyajima and Okajima, 1985). Major advantages of enamel include near absence of organic material with its associated competing EPR signal. Among the disadvantages are preparation-induced signals which interfere with measurement of the main radiation-sensitive signal.

There are two major signals as well as several smaller ones which are induced by mechanical trauma (Desrosiers ef al., 1989). One of these has a L.ande value of $g=2.0020$ and mily be due to mechanically-induced amplification of the main hydroxyapatite signal, or a new signal which is almost exactly superimposed on the radiation-sensitive hydroxyaputite signal. The magnitude of this signal may be the equivalent of several Gy of experimental irradiation. Desrosiers et al. (1989), reported generating this signal by preparing granules with a dental drill.

Generation of the $g=2.0020$ signal can be avoided if a gentler method of sample preparation is used, i.e. grinding with a mortar and pestle (Desrosiers et al., 1989). In this case, a small signal is generated at $g=2.0038$ (Desrosiers et al.. 1989; Pass and Aldrich, 1985: Tatsumi-Miyajima and Okajima, 1985).

Polyakov et al. (1995), recently demonstrated that the magnitude of the $g=2.0038$ signal is increased by

-To whom all correspondence should be addressed. exposure to "Sr beta radiation. Further, their findings indicate the possibility of overestimation of doses with small grains and underestimation with large grains. Their data indicated that at an average grain size of approx. $200 \mu \mathrm{m}$, the effects were canceled.

In an effort to further elucidate the problems associated with mechanical trauma and grain size on enamel, and to extend the work to include gamma irradiation, we tested the effects of gamma rays applied to enamel before and after crushing.

\section{Experimental}

The design of the experiment is shown in Fig. 1. An enamel cap from a molar was weighed and irradialed with $5.6 \mathrm{~Gy}$. The cap was then split into two halves which were weighed and designated as sides $C$ and $H$. named for their state at the time of subsequen irradiation, $\mathrm{H}$ for half and $\mathrm{C}$ for crushed. $\mathrm{C}$ was crushed and sieved into four aliquots of sizes $>250$. 106-250, 75-106 and $<75 \mu \mathrm{m}$. The 75-106 $\mu \mathrm{m}$ aliquots were discarded from both the $H$ and $C$ groups because of insulficient sample. EPR spectrat were then taken for each of the three $C$ alliquots separately. These three aliquots were then recombined into one conglomerate which was then reirradiated to a total of $11.2 \mathrm{~Gy}(5.6+5.6)$. Following this, the grains were resieved hack into the same three aliquots and each was individually. scanned. $H$ was reirradiated prior to crushing. 10 a total of I1.2 Gy. It was then crushed and sieved into the same sizes as was $C$. The thrce aliquots from $B$ were then scanned. The masses of the granules are given in Table 1.

For purposes of mass normilization, we had previously determined the EPR response to enamel 


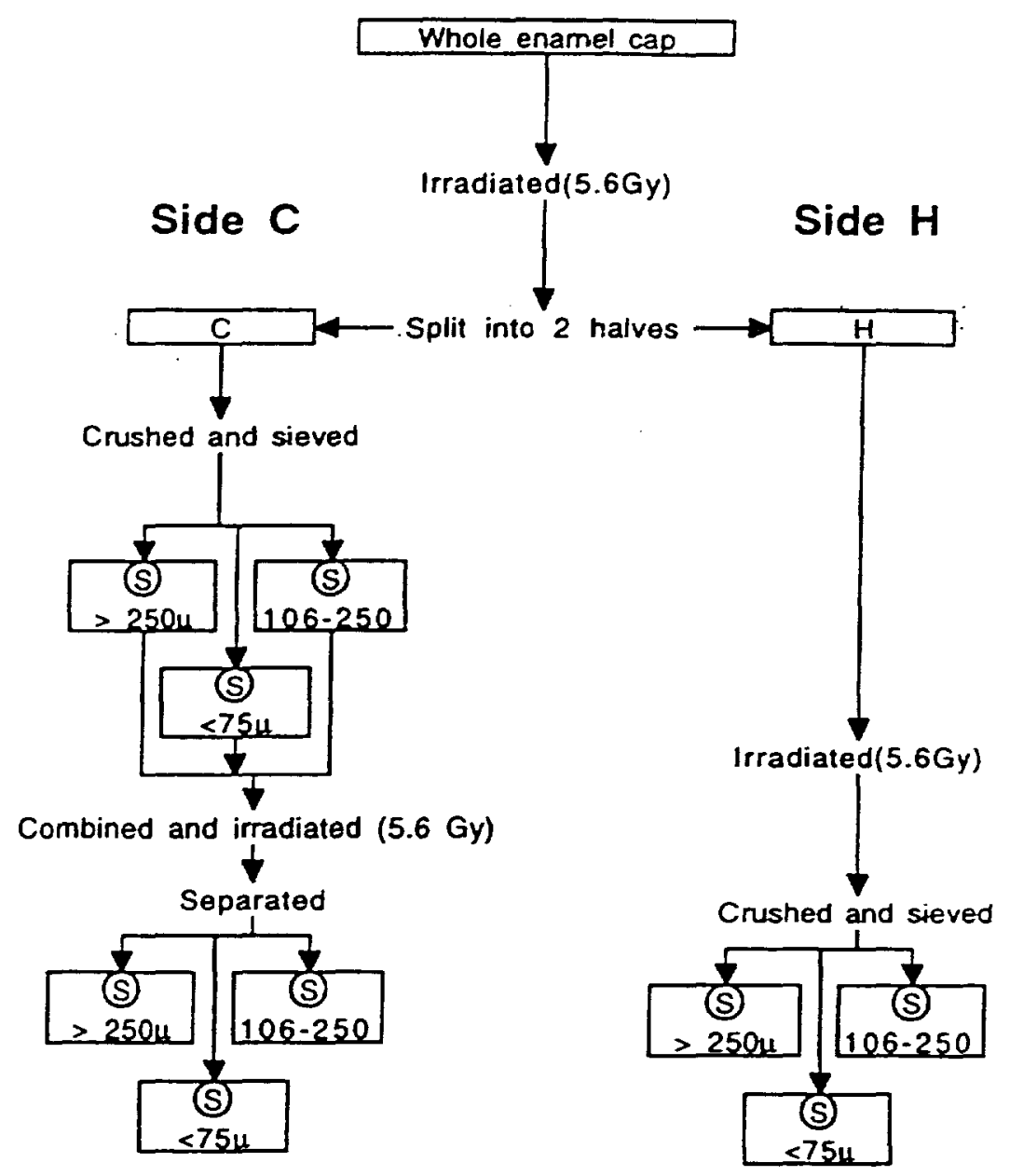

Fig. 1. Experimental protocol. Symbol (encircled S): specimen was sanned at $1 \mathrm{~mW}$ :

granules over a range of $0-100 \mathrm{mg}$ in $5 \mathrm{mg}$ increments. The results were linear with $R=0.990$.

Dose responses were obtained at 5.6 and $11.2 \mathrm{~Gy}$ for the three $C$ aliquots. This was done to test Polyakov's observation that there is a grain-size-sensitivity relationship.

The tooth used in this study was obtained from a routine extraction by the dental clinic of the Salt Lake City FHP (Family Health Plan) Hospital. Records of previous diagnostic $X$-rays were not available. Approximately the apical one-fourth of the tooth was removed with water irrigation using a $4^{*} \times 0.012^{*}$ $(10.2 \mathrm{~cm} \times 0.03 \mathrm{~cm})$ diamond saw blade mounted on a Buchler Isomel saw (Buehler Lid. Lake Bluff. IL

Table 1. Crushed sample musses (mg)

\begin{tabular}{|c|c|c|c|}
\hline Specimen group & $>250 \mu$ & $\begin{array}{c}\text { Grain size } \\
106-250 \mathrm{It}\end{array}$ & $<75 \mu$ \\
\hline $\begin{array}{l}\text { Crushed sample C, Ist scan } \\
\text { Malss }\end{array}$ & 118.47 & 42.21 & 29.03 \\
\hline $\begin{array}{l}\text { Crushed simple C, 2nd scan } \\
\text { Miass } \\
\text { Cruslied simple H }\end{array}$ & 112.14 & $\$ 1.90$ & 27.85 \\
\hline Miss & 103.56 & 65.17 & 52.54 \\
\hline
\end{tabular}

60044). This removed most of the root of the tooth. The remaining dentine was removed with a dental drill using a ball bur. This was practical because the enamel could be visually differentiated from the darker dentine.

The cap was divided in halves using the Buetler Isomet Saw. In this case, an effort was made at cutting which resulted in the specimen spitting neatly in half shortly after cutting began. Crushing was done with an agate mortar and pesile.

The specimens were irradialed with a "Co source in a volume irradiator (US Nuclear. Model GRY. Burbank, Calif.) with a dose rate to hydroxyapatite of $0.22 \mathrm{~Gy} \mathrm{~min}^{-1}$. Calibration of the sourci was done with alanine EPR dosinxters (Albrechl Wivex Messtechnik, Munchen, Germany). The whole and half caps were irradiated in a copper cylinder $(16 \mathrm{~mm}$ o.d. $\times 66 \mathrm{~mm}$ long. wall thickness $2 \mathrm{~mm}$ ). The bottom $32 \mathrm{~mm}$ of the cylinder was filled with a plug of nyion glued in place with epoxy resin. A $45 \mathrm{~mm}$ long rod of nylon was used as a cap. The granules were irradiated in an aluminum holder $(10.7 \mathrm{~mm}$ o.d.. wall thickness of $2.7 \mathrm{~mm}$ ). Confirmation of 


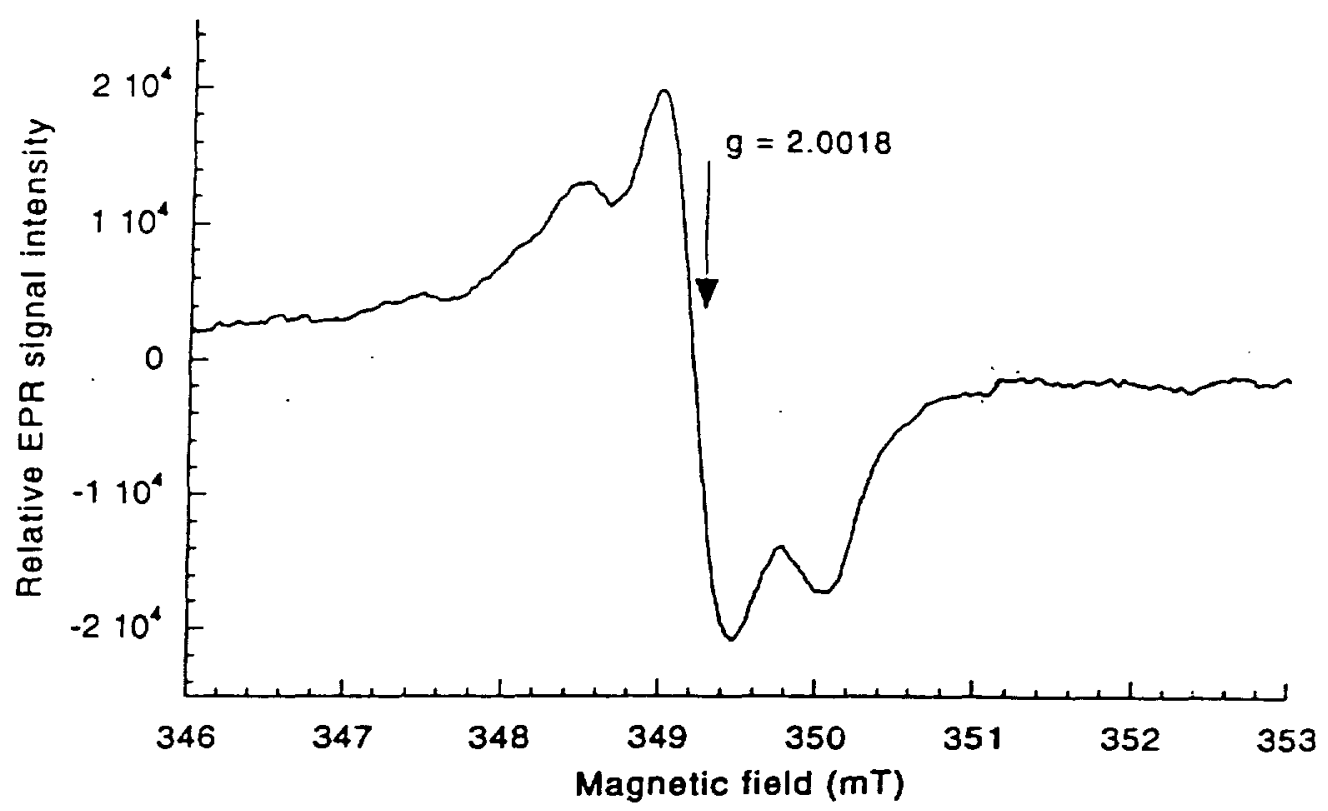

Fig. 2. Typical EPR spectrum for enamet. Post-irradiated specimen. 106-250 $\mu \mathrm{m}$. 40 sueeps. Power $1 \mathrm{~mW}$.

electron buildup and equivalence of absorbed dose to hydroxyapatite with the two capsule configurations was made using a commercial program for modeling dose deposition (PHOTCOEF, AIC Software, Grafton, MA 01519).

The EPR analysis was done using a conventional $X$ band spectrometcr (Bruker Instruments, Billerica, Mass.). Parameters used were power $1 \mathrm{~mW}$, field modulation irequency $100 \mathrm{kHz}$, modulation amplitude $0.5 \mathrm{mT}$. time constant $164 \times 10^{-1} \mathrm{~s}$, receiver gain $1 \times 10^{\circ}$, room temperature. The microwave resonance frequency was approx. $9.7 \mathrm{gHz}$. Scan width was $7.5 \mathrm{mT}$. Weak pitch was used as the reference standalrd Sweep rate was I sweep/0.43 min. Granules $>250 \mu \mathrm{m}$ were scanned twice for 10 sweeps each. 106-250 $\mu \mathrm{m}$ and $<75 \mu \mathrm{m}$ were scanned once for 40 sweeps. excipt for the measurement made on the $106-250 \mathrm{~mm}$ granules at $11.2 \mathrm{~Gy}$ of the $C$ specimens which were scanned three times for 40 sweeps each. Thu powder was placed in a $0.495 \mathrm{~cm}$ o.d. $\times 17.8 \mathrm{~cm}$ long quartz EPR sampling tube (Wilmad Glass. Berna. NJ 08310). All spectra were mass normalized (using a linear mass vs signal size relationship) to a $100 \mathrm{mg}$ sample size. This was done by multiplying the spectral by a factor of 100 divided by the sample's actual mass. The samples were stored at room temperature and low (ambient) humidity.

Measurements of the EPR signals were made peak-to-peak. The peaks were smoothed over $0.01 \mathrm{mT}$. The background signal was not subtracted from the spectra. Statistics were done using standard methods (Spiegel, 1961).

\section{Results}

Figure 2 shows a typical EPR spectrum for enamel.

Figure 3 shows the dose-response of the pustirradiated specimens (first dose to whole cap. second dose to grains). The specimens shown at $5.6 \mathrm{~Gy}$ were irradiated as a whole enamel cap. split into two halves, one of which was crushed and then scanned. The resulting granules were then given an additional dose of 5.6 Gy and rescanned. The granules decrease in sensitivity with decreasing grain size as reported by Polyakov and his coworkers.

Figure 4 shows the dose-response of the preirradiated specimens (both irradiations applied prior to crushing). The points at $5.6 \mathrm{~Gy}$ (samk as in Fig. 3 ) and the $11.2 \mathrm{~Gy}$ (re-irradiation of remaining half cap. $5.6 \mathrm{~Gy}+5.6 \mathrm{~Gy}$ ) were all irradialed before crushing. The sensitivities are similar for all grain sizes. An increasing negative olTset is setn wh decreising graun size.

Polyakov and his coworkers reported that there appeared to be a crossover point at about $200 \mu \mathrm{m}$ where the mechanical signal in large grains enhanced the sensitivity of the hydroxyapatite signal while its eftect in small grains was to decrease the sellsitivity. Figure 5. which is a plot of the estimalled dose to the specimen vs grain size. suggests that the crossover point occurs between 200 and $500 \mu \mathrm{m}$.

\section{Discussion}

The postirradiation study (first dose to whole cap. second dose to grains) confirmed the observations of 


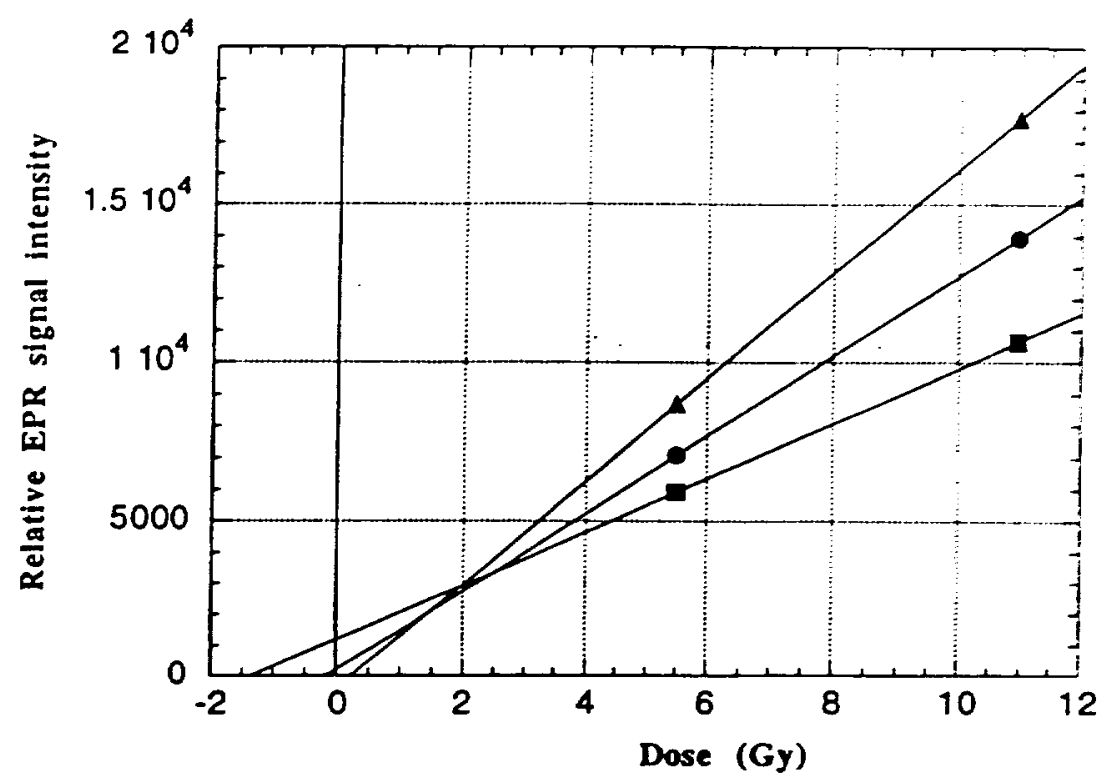

Fig. 3. Dose-response of post-irradiated specimens. The enamel cap was irradiated with $5.6 \mathrm{~Gy}$ and subsequently split in two. One half cap was crushed and the resulting granules were scanned. These are the points at $5.6 \mathrm{~Gy}$. The granules were then given an additional 5.6 Gy and rescanned. These dre the points at $11.2 \mathrm{~Gy}$. Triangles: $>250 \mu \mathrm{m}$. $y=-338+1650 \mathrm{x}$. Circles: $106-250 \mu \mathrm{m} . y=238+1248 \mathrm{x}$. Squares: $<75 \mu \mathrm{m} . \quad y=1191+862 x$. Granules $>250 \mu \mathrm{m}$ were scanned twice for 10 sweeps each ( $\mathrm{SD}=40.3-86.2$ ). 106-250 $\mu \mathrm{m}$ and $<75 \mu \mathrm{m}$ were sanned once for 40 sweeps. except the measurement made on 106-250 $\mu \mathrm{m}$ granules at 12.2 Gy which were scanned three times for 40 sweeps each (SD $=918.2)$.

Polyakov et al. (1995) that there is a decrease in sensitivity of the radiation induced hydroxyapatite signal at $g=2.0018$ with decreased grain size.

We interpret the results of the preirradiation study (both irradiations applied prior to crushing) to mean that the mechanical signal is not affected by irradiation received prior to crushing, and that the samples' sensitivities to radiation applied prior to crushing are likewise not grain size dependent. Although it appears that both methods produce errors in dose estimates depending on the size of the grains being analyzed. that is not necessarily the case since a background zero signal has not been subtracted in either method. In the postirradiated

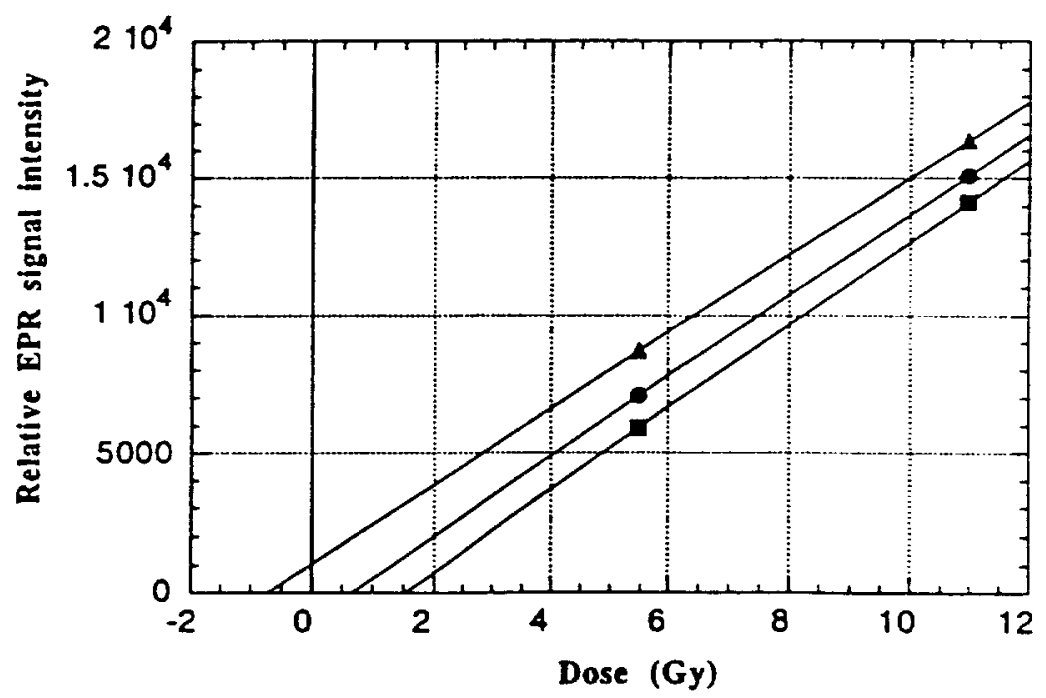

Fig. 4. Dose-response of pre-irradiated specimens. The points at 5.6 and $11.2 \mathrm{~Gy}$ were all irradiated before crushing. Triangles: $>250 \mu \mathrm{m} . y=1029+1401 \mathrm{x}$. Circles: $106-250 \mu \mathrm{m} . y=-925+1260 \mathrm{x}$. Squares: $<75 \mu \mathrm{m} . y=-2281+1496 \mathrm{x}$. The points shown at $5.6 \mathrm{~Gy}$ are the sime as those in Fil. 3 In the case of the 12.2 Gy points: the granules $>250 \mu \mathrm{m}$ were seanned twice for 10 swerps each $(S D=404.5)$ while the $106-250 \mu \mathrm{m}$ and $<75 \mu \mathrm{m}$ granules were seanned once for 40 sweeps. 


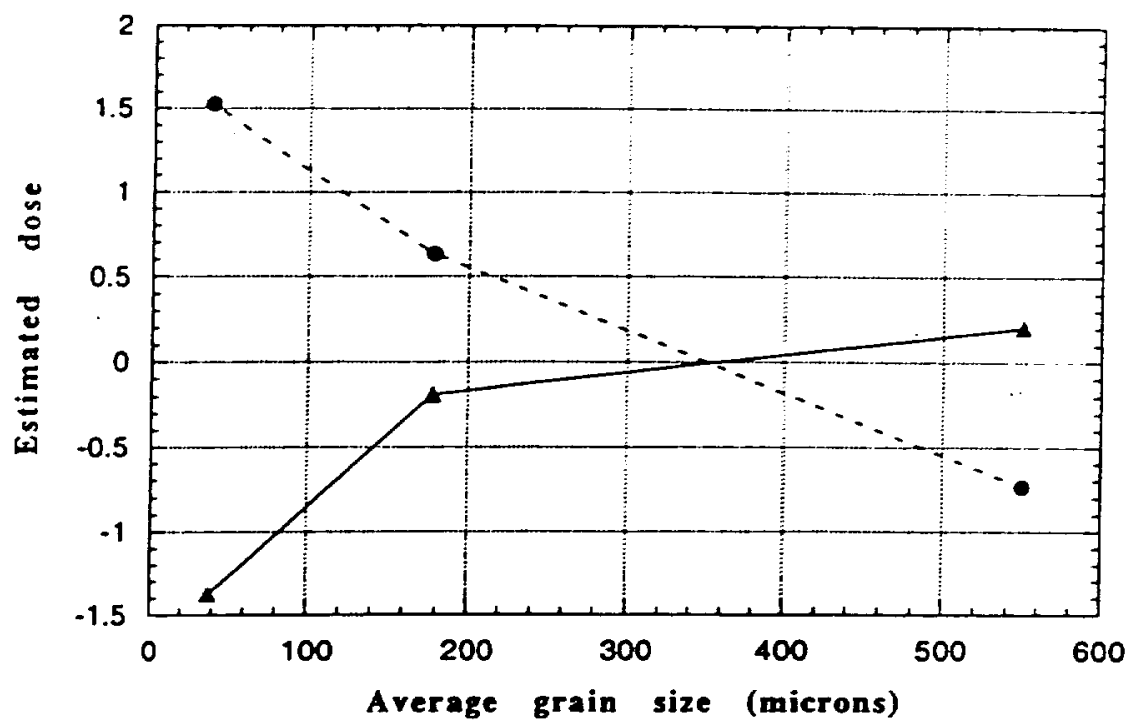

Fig. S. Estimated dose vs average grain size. The estimated grain size was determined by averaging the maxima and minima of each grain fraction. The zero crossover points occur between 300 and $500 \mu m$. Diamonds: estimate from post-irradiation specimens (Fig. 2). $y=0.0027 x-1.14 . R=0.86$. Circles: estimate from pre-irradiated specimens (Fig. 3) $y=-0.00426 x+1.56, R=0.99$.

specimens, subtraction of the background signal appropriate for the grain sizes in question would be expected to produce identical fits for each of the grain sizes since the slopes are similar. The preirradiation study, on the other hand, has errors which are a function of both background and sensitivity change, and subtraction of background signals would not eliminate the errors.

The decrease in sensitivity with decreasing grain size can be explained by postulating either a decrease in the number of, or an increase in competitors of, the radiation-sensitive hydroxyapatite centers. This could be a uniform volume effect with the sniallest grains assumed to have the greatest stress and thus effect, or a surface to volume phenomenon with the surface of all grains affected cqually. The increase of surface to volume ratio with decreasing grain size would then account for the sensilivity dillerence.

Another possible explanation for the effects observed in this study concerns increases in packing density (sample massisample volume) associated with decreasing grain sizes. Although packing density was not examined in this study, such an effect could produce apparent sensitivity changes due to normalization nonlincaritics and could conceivably produce difierential broadening of the mechanical vs the radiation sensitive signal resulting in the grain size dependent offsets observed here. We plan to address this issue in future work.

\section{Conclusion}

We have confirmed the observations of Polyakov et al. (1995) that the sensitivity of hydroxyapatite to radiation is dependent on the size of grains analyzed. Further, our results indicate that preirradiation does not affect the sensitivity of the hydroxyapatite dose response curve. Finally, this experiment suggests that the optimum grain size range for accurate dose estimation would be approx. 200 $500 \mu \mathrm{m}$.

\section{References}

Desrosiers M. F., Simic M. G.. Eichmiller F. C.. Johnston A. D. and Bowen R. L. (1989) Mechanically induced generation of radicals in tooth enamel. Int. J. Rad. Appt Instrum. Ser. A 40, 1195

Grūn R., Beaumont P. B. and Stringer C. B. (1990) ESR dating evidence for carly modern humans al Border Cave in South Africa. Narure 344, 537

Ikeya M., Miki T., Kui A and Hush M (1986) ESK dosimetry of A-bomb radiation using tooth enamel and granite rocks. Rudiat. Prosect. Dosim. 34. 181 .

Ishii $H$. and Ikeya $M$. (199n) An electron spin resonance system for in cilow humin teeth dusimetry Jap. J. Appl. Phys. 29, 871

Nishiwaki Y. and Shimano T. (1990) Lncertuinties in dose estimatation under emergency conditions and ESR dosimetry with human teeth. Radiat Profect. Desim. 34, 295

Pass B. and Aldrich J. E. (1985) Dental enamel as an in cire radiation dosimeter. M/cd. Pha 12305

Polyakov V.. Haskell E.. Kenner G. Huetl G. and Hayes R. (1995) Efrect of mechanically indued background signal on EPR dosimetry of iooth enamel. Radiut. Measure. 00, 000.

Rink W. J. and Schwarcz H. P. (14)4 ) Dose response of ESR signals in tooth enamel. Int. J. Radiut. Appl. Insirum. Part D: Rudiut. Meusure 23, 481 .

Shimano T., Iwasaki M., Miyazawa C.. Miki T.. Kai A. and Ikeya M. (1989) Human tooth dosimetry for gamma-rays and dental $X$-rays using ESR. Afpl. Radiat. Isot 40, 1035 . 
Spiegel M. R. (196i) Theory and Problems of Sturistics. Tatsumi-Miyajims J. and Okajims $S$ (1985) ESR McGraw-Hill. Sun Francisco. Calif.

Stringer C. B. Grün R., Schwarea H. P. and Goldberg P.

(1989) ESR dates for the hominid burial site of Es Skhul dosimetry using human woth enamel in F.SR Desrmi und Dusimusy (lkeya M. and Miki T.. Eds). IONICS.

in Israel. Nature 338, 756.

Tokyo. 


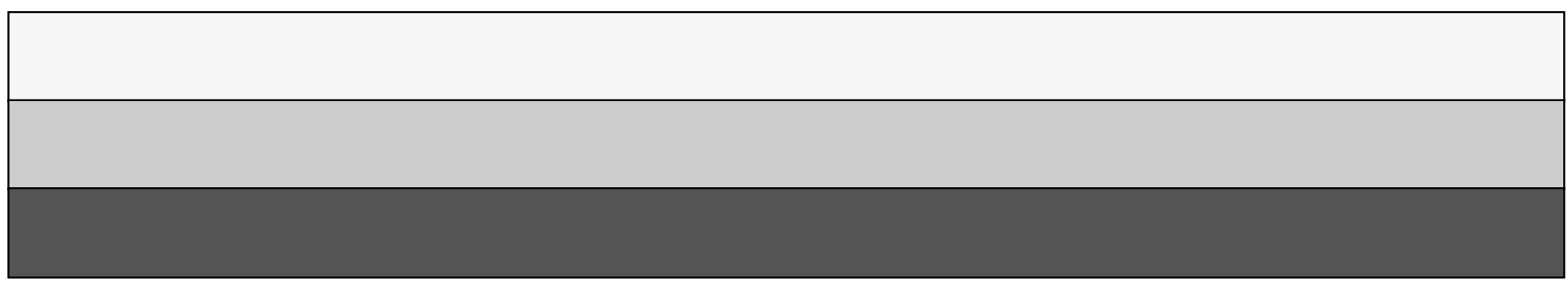

